\title{
Getting the Driver back into the Loop: The Quality of manual Vehicle Control following long and short non-critical Transfer-of-control Requests: TI:NS
}

\author{
Sanna M. Pampel ${ }^{\mathrm{a}}$, David R. Large ${ }^{\mathrm{b}}$, Gary Burnett ${ }^{\mathrm{c}}$, Rebecca Matthias ${ }^{\mathrm{d}}$, \\ Simon Thompson ${ }^{\mathrm{e}}$, Lee Skrypchuk ${ }^{\mathrm{f}}$
}

${ }^{a}+44$ (0)115951 4038, sanna.pampel@nottingham.ac.uk, Human Factors Research Group, Faculty of Engineering, University of Nottingham, NG7 2RD, UK, ORCiD: 0000-0002-3897-6258, https://www.linkedin.com/in/sanna-pampel-5585b712/, @SannaPampel

${ }^{b}+44(0) 115951$ 4040, david.r.large@nottingham.ac.uk, Human Factors Research Group, Faculty of Engineering, University of Nottingham, NG7 2RD, UK, https://www.linkedin.com/in/david-r-largel, ORCiD: 0000-0003-3046-4984

${ }^{c}+44(0) 115951$ 3800, gary.burnett@nottingham.ac.uk, Human Factors Research Group, Faculty of Engineering, University of Nottingham, NG7 2RD, UK

d+44(0) 247656 7237,rmatthia@jaguarlandrover.com, JLR Research, Jaguar Land Rover, Coventry, CV3 4LF, UK, Linked In - https://www.linkedin.com/in/rebeccabartlett-matthias-4953ab23/

e+44(0) 2476151 548, sthom261@jaguarlandrover.com, JLR Research, Jaguar Land Rover Limited, Abbey Road, Whitley, Coventry CV3 4LF, UK, https://www.linkedin.com/in/simon-thompson-69142411/ f+44(0) 247615 1497, lskrypch@jaguarlandrover.com, JLR Research, Jaguar Land Rover, Coventry, CV3 4LF, UK, ORCiD: 0000-0002-9619-6161

Dr Sanna M. Pampel is a Research Fellow in the Human Factors Research Group in the University of Nottingham. She is particularly interested in Automotive Human Factors and has worked with mental models, driving styles as well as novel human machine interfaces, such as digital mirrors, driver state measuring, automated driving, and augmented reality technology, 
mostly in collaboration with car manufacturers. Prior to her $\mathrm{PhD}$ in the University of Leeds she gained 4 years' worth of experience in the IT industry.

Dr David R. Large is a Research Fellow with the Human Factors Research Group at the University of Nottingham. Since returning to academia and gaining his PhD in 2013, David has been involved in a broad range of industry, UK and EU funded projects primarily concerning the design, evaluation and acceptance of novel and emerging in-vehicle interfaces and systems for both road and rail transport, and the development of experimental techniques to support this work.

Professor Gary Burnett holds a chair in Transport Human Factors within the Faculty of Engineering at the University of Nottingham. He has over 25 years' experience in Human Factors research and development relating to advanced technology within road-based vehicles. His work addresses key safety, usability, and acceptance issues for advanced in-car systems and Human-Machine Interfaces (HMIs), and he is particularly concerned with the assessment of driver distraction.

Rebecca Matthias works at Jaguar Land Rover in the Research Department. As a Human Factors Group Leader in Capability Research, Rebecca's work focuses on the human factors aspects of automated driving, in particularly occupant interaction with the highly automated vehicle and vehicle behaviour.

Simon Thompson works at Jaguar Land Rover in the Research Department. As a Human Machine Interface Specialist, Simon focuses on technical aspects such as engineering, human factors, and psychological factors. Simon is responsible for research projects and activities including: designing system-driver interfaces; providing HMI and ergonomics guidance to engineers; competitor benchmarking and assessment; designing, organising and conducting user trials; reviewing and creating internal HMI standards; and developing technology and feature HMI strategies.

Lee Skrypchuk works at Jaguar Land Rover in the Research Department. As a Human Machine Interface Technical Specialist, Lee focuses on technical aspects such as engineering, human factors, and psychological factors. Lee has led projects including head-up display, driver monitoring, and gesture control, and holds responsibility for research roadmaps in this area. 


\title{
Getting the Driver back into the Loop: The Quality of manual Vehicle Control following long and short non-critical Transfer-of-control Requests: TI:NS
}

\begin{abstract}
Specific vehicle automation use-cases such as traffic jams will be the first level 3 functions on the market. When the 'traffic jam pilot' nears its limits in noncritical situations, control needs to be handed back to the driver, enabling appropriate situation awareness (SA) and vehicle handling. According to previous research, operational vehicle stabilisation can be achieved within a transfer-of-control (TOC) of a few seconds in simple traffic environments, but tactical level decisions benefit from longer hand-over times. To date, the effects of non-critical TOCs have not been studied using set time frames. To investigate the impact of short (unplanned, 5 seconds) and long (planned, 50 seconds) TOC requests, while playing/not playing an engaging tablet game, a simulator experiment was conducted with 16 participants. Comparisons of the 60 -secondperiod of manual driving following automation suggest better longitudinal vehicle control as well as more appropriate SA following the long TOC request, and automation periods without the game. However, following no engaging game, lateral performance was worse during the first 10 seconds of manual driving. Control-level visual search patterns did not change with TOC time or the game. Future research needs to consider support for drivers' SA maintenance and readiness to drive following high automation.
\end{abstract}

Keywords: automated driving; driving simulator; transfer of control; vehicle control; eye tracking; driver behaviour

\section{Relevance to human factors / ergonomics theory}

Vehicle automation use-cases such as traffic jams will be the first level 3 functions on the market. When the 'traffic jam pilot' nears its limits, control needs to be handed back to the driver. Although critical situations are crucial to assess, situations that that do not require responding to a time-critical event have received little attention in the literature. Such situations are important, because failure to respond appropriately could lead to unstable driving behaviours such as poor lane keeping and insufficient visual scanning. 
In addition, because the choice of the takeover time is usually left to the driver, longer and shorter transfer times have not yet been assessed in isolation. The present study investigated vehicle control and visual behaviour following short (5 seconds) and long (50 seconds) transfers of vehicle control with fixed time periods. In half of the drives, participants were subjected to a tablet game to create realistic out-of-the-loop experiences. The findings aid the understanding of regaining situation awareness and vehicle control on the tactical and control levels following level 3 automated driving.

\section{Introduction}

\section{Background}

While engaging with level 2 automation, drivers are expected to constantly monitor the environment and be prepared to intervene at any time (Society of Automotive Engineers 2014). However, in reality, it is likely that machine operators become 'Out Of The Loop' during automation (OOTL, Endsley and Kiris 1995). When the person in the driver's seat is OOTL, they enter a state of passive information processing and a loss of Situation Awareness (SA, Endsley and Kiris 1995). Banks et al. (2018) explored the concept of OOTL by observing Tesla drivers on the road and found that people tend to over-trust, disengage, and even perform non-driving activities with this level 2 vehicle. The expectations that drivers remain 'in the loop' and fully aware of the system's limitations, led the National Highway Traffic Safety Administration (NHTSA 2017) to declare the cause of the fatal Tesla crash in California in 2016 to be 'driver error'. There is a history of assigning the causes of accidents on an individual's loss of SA, but Dekker (2015) criticised this approach as too limiting. Salmon et al. (2016) analysed the Air France 447 crash from 2009 using a distributed SA paradigm. The official accident investigation report concluded that the aircrew were initially not aware of the aircraft's 
state and thus unable to intervene appropriately. However, the authors pointed out how the entire system, beginning with the non-human airplane's systems, lost SA. Hence, to improve safety and performance, it is crucial to take the automation as well as the driver into account.

Automotive technology will soon be able to provide cars with level 3 automation capabilities, which do not require constant monitoring during automated periods. Level 3 automation will initially be limited to well-defined scenarios such as congestion, e.g. slow-moving traffic jams on the motorway. Such functions relieve the driver of the more tedious and tiring parts of driving. As the driver is not required to monitor the driving scene during the automated mode, they are able to benefit from the automation, and also engage in a limited range of non-driving activities (cf. Jamson et al. 2013). This means that they may disengage from the driving task to a large extent, and become OOTL.

When transitioning back to manual driving, critical elements of the SA need to be regained. Immediately important are the awareness of the system state, e.g. whether the vehicle automation is activated, and the spatial awareness of one's own as well as other road users. Soon the driver needs to ensure the awareness of other situational elements such as temporal changes in the environment and the driver's current relation to their navigational goals (cf. Matthews et al. 2001). Gold et al. (2016) found that a Transfer Of Control (TOC) in dense traffic conditions led to longer reaction times, suggesting that additional time was needed for sufficient visual scanning, comprehension and projection, the building blocks for SA (Endsley 1995), and thus informed decision making. Besides the information processing aspect, OOTL also includes a strong physical, or sensory-motor control, component (Endsley and Kiris 1995). To support this, it was found that, even when monitoring the driving scene, 
automation leads to disengagement from the driving task and thus performance decrements after TOC (Louw, Merat, and Jamson 2015). The lowered performance is apparently independent of the level of cognitive task engagement (c.f. Gold et al. 2016), further supporting the importance of the physical driving engagement.

In order to understand human behaviours, they have been described in hierarchical models, such as Rasmussen's skills, rules and knowledge taxonomy (Rasmussen 1983). Michon (1985) described a similar taxonomy for the driving domain, with strategies on the highest level, tactical decision-making on the manoeuvring level, and vehicle control behaviours on the lowest, 'automatic' level. Automatic behaviours are skills that have been trained sufficiently, so they can be executed without conscious attention (Boer and Hoedemaeker 1998).

During the TOC to the driver, human control needs to be regained at all three levels of Michon's hierarchy. For this to be achieved, the driver needs to perceive and process the necessary environmental input in order to build a sufficient level of SA, be able to make appropriate decisions and carry out the necessary actions.

On the strategic level, drivers make conscious choices, e.g. they orientate themselves and make plans. Here, drivers mainly need to make future projections in order to decide on the next steps on their route, e.g. choosing an upcoming exit, but also perceive and comprehend their situation, e.g. the current location (Matthews et al. 2001). This strategic level also includes the awareness of the current automation level, e.g. whether it is safe to engage in secondary tasks or to sleep, which is crucial for the safe handling of the vehicle. A small number of studies involving automation and TOC strategies include the strategic level, where drivers orientate themselves on the road and become conscious of the next steps on the route. Damböck et al. (2012) measured 
performance on this level with moving into the correct lane, according to the direction the participants were directed to while approaching a motorway junction.

On the tactical level, drivers perceive environmental cues, comprehend and judge the current situation, and might perform a limited amount of projection (Matthews et al. 2001; Ward 2000). They scan the environment, check the mirrors and decide which skill to activate or deactivate on the control level, e.g. whether the driver should coast to reduce the distance to a car in front or step on the brake. Goodrich and Boer (2003) measured such possible brake responses to explore the boundaries between acceptable and unacceptable safety margins to a front car while using an Adaptive Cruise Control (ACC). A tactical action can also include placing the hands on the steering wheel following a TOC to manual, although this action does not require conscious judgements or decisions (Gold et al. 2016). Several TOC studies consider the tactical level such as visual scanning patterns, the orientation of the driver in the immediate road environment by fixating on other road users as well as decisions about manoeuvres. Louw et al. (2015) forced drivers in a simulator study to decide about avoiding an obstacle by changing lanes. In comparison to manual driving, the responses to the obstacle were delayed, and more aggressive after brief periods of automation, whether the drivers completed a distracting task or not. Nevertheless, when the participants were observing the driving scene during the automation, their responses were more calculated with less lateral acceleration. When Eriksson and Stanton (2017b) asked drivers to read a magazine during automation, manual reactions following a takeover request were slower, compared to a non-distracted condition. Hence, there is evidence that drivers observe the road environment during automation, especially when tactical-level considerations such as reactions to potential critical events are important. Findings by Jamson et al. (2013) support this, and that drivers are more willing to 
disengage from control-level activities. Kircher et al. (2014) measured tactical driver interventions when different types of automation reached their system limits. As expected, higher levels of automation, with the highest being comparable to level 2 , led to more reliance on the system and acceptance of lower safety margins than during manual driving. Nevertheless, drivers observed the road scene during automation and intervened when necessary, although establishing sufficient SA in critical situations required more visual search following higher automation. Nevertheless, longer TOC times are likely to be beneficial for the tactical level. Damböck et al. (2012) tested performance with an approaching lane closure, which was responded to with more timely lane changes after longer TOCs, specifically 8 seconds instead of 4 seconds. Automatic, or control-level, driving tasks such as speed regulation and lane keeping are performed on a subconscious level, even by drivers with relatively little driving experience (Young and Stanton 2007). Most of the required SA builds upon perceptions through hazard detection, immediate haptic feedback of the pedals and steering wheel, or the immediate visible results of the driver's input such as a change in speed or direction. Some comprehension, for example hazard evaluation and projection, such as predictions of the lane position, are also performed on this level (Ward 2000). The majority of studies investigating TOC situations regard lower level behaviours, which mostly describe a driver's ability to stabilise the vehicle. Reasons are that automation features have first been developed for this level, and that this is the most important level to focus on to avoid crashes as a result of unstable driving (Damböck et al. 2012). Relevant measures of control-level performance can include speed and lane position (Merat et al. 2014), and whether a crash occurs in a simulated environment (Damböck et al. 2012). Establishing acceptable manual driving performance following a period of automation, especially the lateral stabilisation of the vehicle, has been shown 
to be quicker when drivers expect the TOC (Merat et al. 2014). However, reading a newspaper during the automation had no significant effect on lateral stabilisation after TOC, as Eriksson and Stanton (2017b) found by observing the variation of the steering wheel angle. With regards to the time required for the TOC, skill-level performance tends to be sufficiently good, even with four seconds between the onset of the request and the end of the automation (Damböck et al. 2012).

During critical situations that require a TOC to the driver, it is most important that the driver takes over the driving task on the control level within a very short amount of time to react to a potential event and prevent a crash. Although critical situations are crucial to assess, situations that do not require a response to a time-critical event are important too, as failure to respond appropriately could potentially lead to unstable driving behaviours such as poor lane keeping and insufficient visual scanning. Hence, there is a need to understand the behaviour of drivers following durations of automated driving that could in theory enable OOTL driving. One of the possible noncritical scenarios is when drivers choose to intervene, e.g. to perform a manoeuvre such as a lane change (Madigan, Louw, and Merat 2018) or because they enjoy driving manually. Non-critical TOCs initiated by the vehicle can include a variety of unplanned and planned situations. Unplanned situations include a sudden end of the automation conditions, such as discontinuing lane markings or traffic dispersal in the case of trafficjam assist systems, while planned TOCs can theoretically be much longer, e.g. when a known motorway exit approaches. With longer TOCs, the driver might have the additional possibilities to gain more comprehensive SA, as van der Heiden et al. (2017) found when presenting warnings 20 seconds before handover. This way, drivers may be able to better prepare for the higher levels of the driving task before the automation ends. If unplanned, the non-critical manual conditions can provide time to initially 
stabilise the vehicle before making decisions on the tactical and strategic levels. Few researchers have considered non-critical transitions from high automation to manual (e.g. Eriksson and Stanton 2017b; Madigan, Louw, and Merat 2018; Naujoks, Mai, and Neukum 2014). However, because they let the drivers choose the time of the takeover in these studies, following the vehicle's request, the effects of longer and shorter TOC cannot be assessed in isolation.

\section{The current Study}

The present study focussed on non-critical TOC from level 3 automation to manual driving in order to expand theoretical knowledge of cases that can be expected due to system limitations. When drivers are aware of the environment's as well as the vehicle's state, they may be better prepared for manual interventions, as was shown with ACC (Seppelt and Lee 2007) and level 2 systems (Beller, Heesen, and Vollrath 2013), e.g. by observing the road scene during automation and building appropriate SA after a TOC. During our investigation, both long (planned) and short (unplanned) TOCs are of interest. In theory, lengthy TOCs provide time for the driver to acquire SA and prepare tactical interventions. Thus, drivers may be more ready to assume control of their vehicle, while taking into account other nearby vehicles. Following short TOCs, drivers may prioritise control-level driving behaviours to first stabilise their vehicle, and subsequently prepare for decisions at the tactical and strategic levels, for example by increasing visually scanning of the environment including the relevant HMI. Although drivers would be expected to be able to override the automation state in practice as they respond to a take-over request (i.e. resume manual control immediately, rather than wait for the hand-over period to expire), it was decided to control the length of the TOC for this study's purpose (i.e. remove this capability from drivers) to isolate the effects of the transition time. The 'short' TOC time was therefore set to 5 seconds, to correspond with 
the critical transition times in previous studies, while not undermining typical reaction times (e.g. Damböck et al. 2012; Gold et al. 2016; Zeeb, Buchner, and Schrauf 2016). The 'long' TOC was set at 50 seconds; this was deemed to be an appropriate duration (following consultations with industry experts), as it can represent a situation in which a known motorway exit is approached (cf. Roncoli, Papageorgiou, and Papamichail 2015). This time frame may also be sufficiently long for the driver to fully prepare for manual driving. It was hypothesised that long TOCs led to more efficient visual scanning due to more comprehensive SA as well as better driving on the tactical level and thus higher speeds to adjust to the changing traffic conditions.

In addition to short and long TOC, a distracting secondary task was added to the current study design to overtly disengage drivers visually and cognitively from the driving task during the automation. In this way, it could be assessed to which degree drivers observed the environment during the automation, when not distracted. Thus, it was also hypothesised that the distraction task may affect vehicle stabilisation as well as visual search, due to deficits in SA on the control and tactical levels.

Because it has been found that the stabilisation after assuming manual control may take up to 40 seconds (Merat et al. 2014), it was particularly interesting to investigate the effects of the interventions in the first 60 seconds after the end of the automation. Especially for eye tracking measures, 60 seconds provide a useful window (Victor 2005). Investigating this period can inform on the time it takes drivers to resume stable control of the vehicle. For more detail about how the measures of interest change within this time frame, it was decided to employ a detailed analysis and comparison by splitting the one-minute period into 5.0-second time bins (cf. Merat et al. 2014). 


\section{Materials and Methods}

\section{Design}

A three-way, within-subjects design was applied. Two comparable HMIs were utilised one warned the driver for a period of 5 seconds ('short'), and the other one for 50 seconds before hand-over ('long'). The second independent variable, Task, concerned whether drivers were distracted or not when a takeover request was made. This was manipulated as a within-subjects variable, such that each participant undertook two drives for each HMI, one whilst being encouraged to engage with a tablet game while the car was in an automated mode ('distracted'), and one where they are motivated to maintain vigilance with the system monitoring task ('non-distracted').

The third factor, Time, was used in order to consider the changes in the stabilisation of driving after hand-over during the first minute of manual driving. Therefore, these 60 seconds were split into 12 sections of 5 second each.

\section{Transfer-of-control Conditions}

Both TOC conditions employed in the study were non-critical. The first one was referred to as 'short' HMI, which would be issued due to an unanticipated system limit event e.g. loss of lane markings or traffic dispersal. For the purposes of this study, such TOC requests were provided with 5 -seconds notification. For predictable system limit events, the vehicle approaches a part of the route that does not support automated driving, such as exiting from the motorway. In this case, TOC requests are provided 50seconds prior to TOC (referred to as 'long' HMI). In both cases, the TOC HMI remained on display for the first 5 seconds of the manual drive, before returning to the usual manual driving interface. 


\section{Driving Scenarios}

The driving scenario consisted of a busy UK motorway. The participants began the route with about one minute of manual driving, during which the traffic slowed down to $40 \mathrm{mph}$, turning into a moving traffic jam. The automated part of the drive took place while in this congestion. For the purpose of the study, the 'automation' followed the front car at a constant speed of $40 \mathrm{mph}$ (in practice, traffic-jam assist systems would rely upon the presence of other road users in the host vehicle's proximity, as well as lane mediation lines, to determine control actions). Just before the automation stopped, all surrounding traffic dispersed ensuring that it was possible to measure the participants' natural acceleration behaviour and control actions. During the display of the TOC, before the automation was switched off, the participant vehicle maintained its speed of $40 \mathrm{mph}$. It was not possible for the participant to intervene before the end of the TOC period.

\section{Dependent Variables}

Measures included behavioural measures (driving performance) and visual behaviour and are listed in Table 1.

Table 1 Dependent variables

\begin{tabular}{|l|l|}
\hline Measure & Description \\
\hline Speed & $\begin{array}{l}\text { Mean, minimum and standard deviation of speed, measured in } \\
\text { mph }\end{array}$ \\
\hline Lateral stability & The standard deviation of lateral lane position, measured in feet \\
\hline $\begin{array}{l}\text { Mean fixation } \\
\text { duration }\end{array}$ & $\begin{array}{l}\text { The mean was computed using the durations of the fixations from } \\
\text { the previous } 10 \text { seconds to the future } 10 \text { seconds }\end{array}$ \\
\hline $\begin{array}{l}\text { Number of } \\
\text { fixations on HMI }\end{array}$ & $\begin{array}{l}\text { The fixations from the eye tracking data were coded into areas of } \\
\text { interest. For this measure, the number of fixations on the transfer- }\end{array}$ \\
\hline
\end{tabular}




\begin{tabular}{|l|l|}
\hline Percent road centre & $\begin{array}{l}\text { of-control HMI was used } \\
\text { vertically around the mean fixation point). The percentage refers to } \\
\text { all fixations in the time period ranging from the previous } 10 \\
\text { seconds to the future } 10 \text { seconds (cf. Victor, Harbluk, and } \\
\text { Engström 2005) }\end{array}$ \\
\hline Spread of search & $\begin{array}{l}\text { Horizontal spread of eye movements during driving: the standard } \\
\text { deviation of the mean horizontal positions (in degrees, }{ }^{\circ} \text { ) of the } \\
\text { fixations within the time period ranging from the previous } 10 \\
\text { seconds to the future 10 seconds(Chapman and Underwood } 1998 ; \\
\text { Crundall and Underwood 1998) }\end{array}$ \\
\hline
\end{tabular}

\section{Apparatus}

The study was undertaken using a medium-fidelity driving simulator, comprising a matte black right-hand drive Audi TT within a $270^{\circ}$ curved screen setup, see Error!

Reference source not found.. A custom driving scenario, created using STISIM

Version 3 software, was projected onto the screen via three high-definition overhead projectors.

Throughout the study, participants wore SMI eye-tracking glasses (ETG) to capture eye movements (with subsequent analysis focussing on visual behaviour after each TOC).

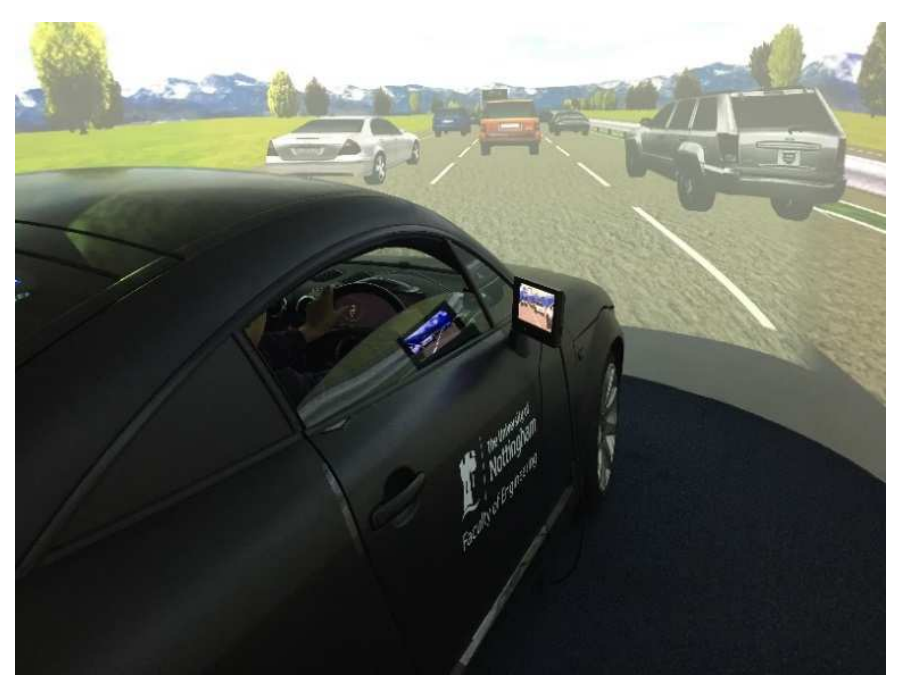


Figure 1

\section{Participants}

Sixteen experienced and active drivers were recruited to take part in the study.

Participants were self-selecting volunteers who responded to advertisements placed around the University of Nottingham campus, and primarily comprised staff members. Participants provided written informed consent before taking part and received a shopping voucher as compensation for their time. The participant base consisted of 9 male and 7 female drivers, with a mean age of 39 years $(\mathrm{SD}=9.7$ years). They possessed a driving licence for an average of 15 years $(\mathrm{SD}=10.4$ years $)$ and were currently driving a distance of 5,000 to 10,000 miles each year.

\section{Procedure}

At the start of the session, the participants were told that, during automation, they would not be required to permanently monitor the system, but may be requested to resume manual control at some point during the drive: furthermore, if this happened, they would be provided appropriate notice. They were also encouraged to do what came naturally to them.

During all drives, participants began driving manually and were instructed to remain in lane 2 (middle lane), although their speed and manoeuvrability was severely restricted by the presence of surrounding traffic. Automated control was activated (at $40 \mathrm{mph}$ ) shortly into the drive at the request of the participant, who spoke aloud a voicecommand: "start automated driving". This initiated a 2.0-second hand-over transition to automated control (although this was in fact activated by the experimenter in a separate control room using a Wizard-of-Oz approach). 
At a different interval during each of the four automated drives (to avoid anticipation), drivers were presented with a TOC request. Following the hand-over, drivers drove manually for at least one minute, before being instructed to bring the vehicle to a controlled stop. Prior to the episode of manual driving, surrounding traffic dispersed to avoid its presence impacting on driver behaviour.

Drivers experienced each HMI ('short' and 'long') during their visit in both a 'distracted' and a 'non-distracted' state. The distraction task was an immersive game (Tetris) on a tablet, engaging the participant in a visual, manual and cognitive manner. The purpose of the game was to distract the driver during the automation, preventing them from observing the road scene. For this, they were encouraged to achieve a score that was as high as possible. The study session, with four drives for each participant, lasted approximately 2.5 hours. All participants were reimbursed $£ 25$ (GBP) in shopping vouchers as compensation for their time.

\section{Data Analysis}

Two analyses were performed for the driving and eye tracking measures. The first analysis compared the first 60 seconds after the hand-over, split into 5-second bins, with each other. The 3-way within-subjects Analysis of Variance (ANOVA) included the factors HMI (short vs. long), Task (distracted vs. non-distracted) and Time (12 levels: the 5-second sections of the first minute of manual driving). Where a significant main effect occurred for Time, post-hoc pairwise comparisons were consulted for the adjacent sections. These were Bonferroni-corrected, based on 11 comparisons.

Additionally, a 2-way repeated measures ANOVA with the factors HMI (short, long) and Task (non-distracted, distracted) was conducted with the total values for the first 60 seconds following the hand-over of the vehicle control to the driver. 
Where the conditions for parametric tests were not met for the majority of conditions, according to the Kolmogorov-Smirnov test and a visual inspection of histograms, a Friedman test was employed for identifying main effects, and the Wilcoxon signed-rank test with Bonferroni corrections was used for subsequent pairwise comparisons.

\section{Results}

\section{Behavioural Measures}

\section{Mean Speed}

The ANOVA resulted in two significant main effects. The first effect occurred for HMI $\left[F(1,15)=11.687, p=.004, \eta^{2}=.438\right]$, where it was found that the 5-second HMI led to a lower mean speed $(M=60.68, S D=6.65)$ compared to the long HMI $(M=63.93, S D$ $=9.95)$. The second effect occurred for Time $\left[F(3.860,57.893)=111.482, p<.001, \eta^{2}=\right.$ .881]. Pairwise post-hoc comparisons assigned significant increases in speed to the $1-5 \mathrm{~s}$ and 5-10 s sections $(\mathrm{p}<.001)$, the $5-10 \mathrm{~s}$ and $10-15 \mathrm{~s}$ sections $(\mathrm{p}<.001)$, as well as the sections from $10-15 \mathrm{~s}$ and $15-20 \mathrm{~s}(\mathrm{p}<.001)$.

An HMI*Time interaction effect $\left[F(3.635,54.526)=2.727, p=.043, \eta^{2}=.154\right]$ showed that the speed was higher for both long HMI conditions, whether distracted or not, with diminishing differences towards the end of the first minute of manual driving, see Error! Reference source not found..

The comparison between the conditions, using the first 60 seconds following the hand-over, resulted in a significant effect for $\operatorname{HMI}\left[\mathrm{F}(1,15)=11.683, \mathrm{p}=.004, \eta^{2}=\right.$ .438], because the long HMI resulted in a mean speed of $63.9 \mathrm{mph}$, as opposed to 60.7 mph with the short HMI. There was no effect for Task $(\mathrm{p}=.678)$ and no HMI*Task interaction effect $(\mathrm{p}=.503)$. 


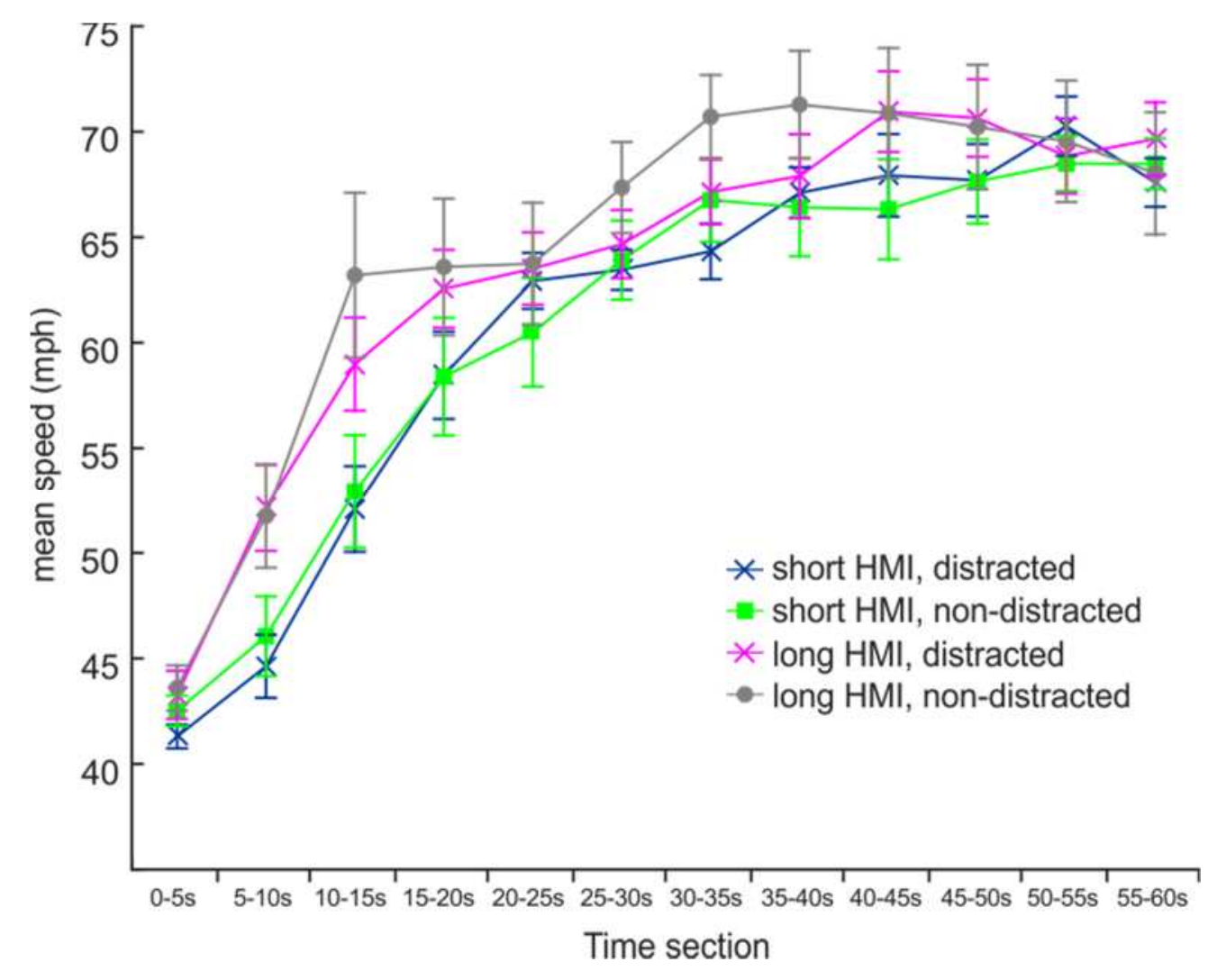

Figure 2

\section{Minimum Speed}

The ANOVA resulted in two significant main effects. The first effect occurred for HMI $\left[F(1,15)=10.263, p=.006, \eta^{2}=.406\right]$. The 5-second HMI led to a lower minimum speed $(\mathrm{M}=37.57, \mathrm{SD}=5.41)$ compared to the 50-second HMI $(\mathrm{M}=38.32, \mathrm{SD}=4.40)$. The second effect occurred for Time $\left[\mathrm{F}(3.455,51.831)=143.887, \mathrm{p}<.001, \eta^{2}=.906\right]$. Pairwise post-hoc comparisons assigned the differences to increases from the 1-5 s to 5$10 \mathrm{~s}(\mathrm{p}<.001)$, the $5-10 \mathrm{~s}$ to $10-15 \mathrm{~s}$ sections $(\mathrm{p}<.001)$, from the section $10-15 \mathrm{~s}$ to $15-$ $20 \mathrm{~s}(\mathrm{p}<.001)$, and then again for $25-30 \mathrm{~s}$ to $30-35 \mathrm{~s}(\mathrm{p}=.022)$. An HMI*Time effect interaction effect $\left[F(3.486,52.284)=3.125, p=.027, \eta^{2}=.172\right]$ confirmed the finding of the analysis of the mean speed. For the first 50 seconds after hand-over, the minimum speed was higher after the drivers were alerted with the long HMI. 
The comparison between the conditions, using the first 60 seconds of manual driving, resulted in no significant effect main for HMI $(\mathrm{p}=.384)$. There was no effect for Task $(p=.779)$ and no HMI*Task interaction effect $(p=.958)$.

\section{Standard Deviation of Speed}

The analysis resulted in a main effect for Time $\left[\mathrm{F}(5.501,82.508)=3.286, \mathrm{p}=.007, \eta^{2}=\right.$ .180]. Post-hoc comparisons assigned this effect solely to a significant increase from $2.32 \mathrm{mph}(\mathrm{SD}=.84)$ to $3.16 \mathrm{mph}(\mathrm{SD}=.84)$ in the $1-5 \mathrm{~s}$ to the $5-10 \mathrm{~s}$ periods respectively $(p=.022)$. The analysis of the mean values for the first 60 seconds did not produce significant effects (HMI: $\mathrm{p}=.576$, Task: .843, HMI*Task: $\mathrm{p}=.567$ ).

\section{Standard Deviation of lateral Lane Position}

The standard deviation of lateral lane position data was not normally distributed and so non-parametric tests were conducted. Friedman tests showed a significant main effect for the short HMI when not distracted $\left[\chi^{2}(11, \mathrm{~N}=16)=29.067, \mathrm{p}=.002, \varphi=1.348\right]$. Post-hoc Wilcoxon signed-rank tests assigned the main effect to a decrease from 1-5 seconds and 5-10 seconds $(\mathrm{p}=.011)$, and another stabilisation from 15-20 seconds to 20-25 seconds ( $p=.036$ ), visible in Error! Reference source not found.. The Friedman tests did not show significant main effects for the short HMI when distracted $(\mathrm{p}=.246)$

The long HMI produced a main effect when not distracted: $\left[\chi^{2}(11, \mathrm{~N}=16)=\right.$ 35.596, $\mathrm{p}<.001, \varphi=1.492]$. Post-hoc tests showed a decrease from 1-5 seconds and 510 seconds $(\mathrm{p}=.036)$. Despite a main effect for the distracted long HMI condition $\left[\chi^{2}(11, \mathrm{~N}=16)=24.356, \mathrm{p}=.011, \varphi=1.234\right]$, post-hoc tests showed no differences. Regarding the comparison of the first 60 -second means, the Friedman test did not highlight any significant effects $(\mathrm{p}=.149)$. 


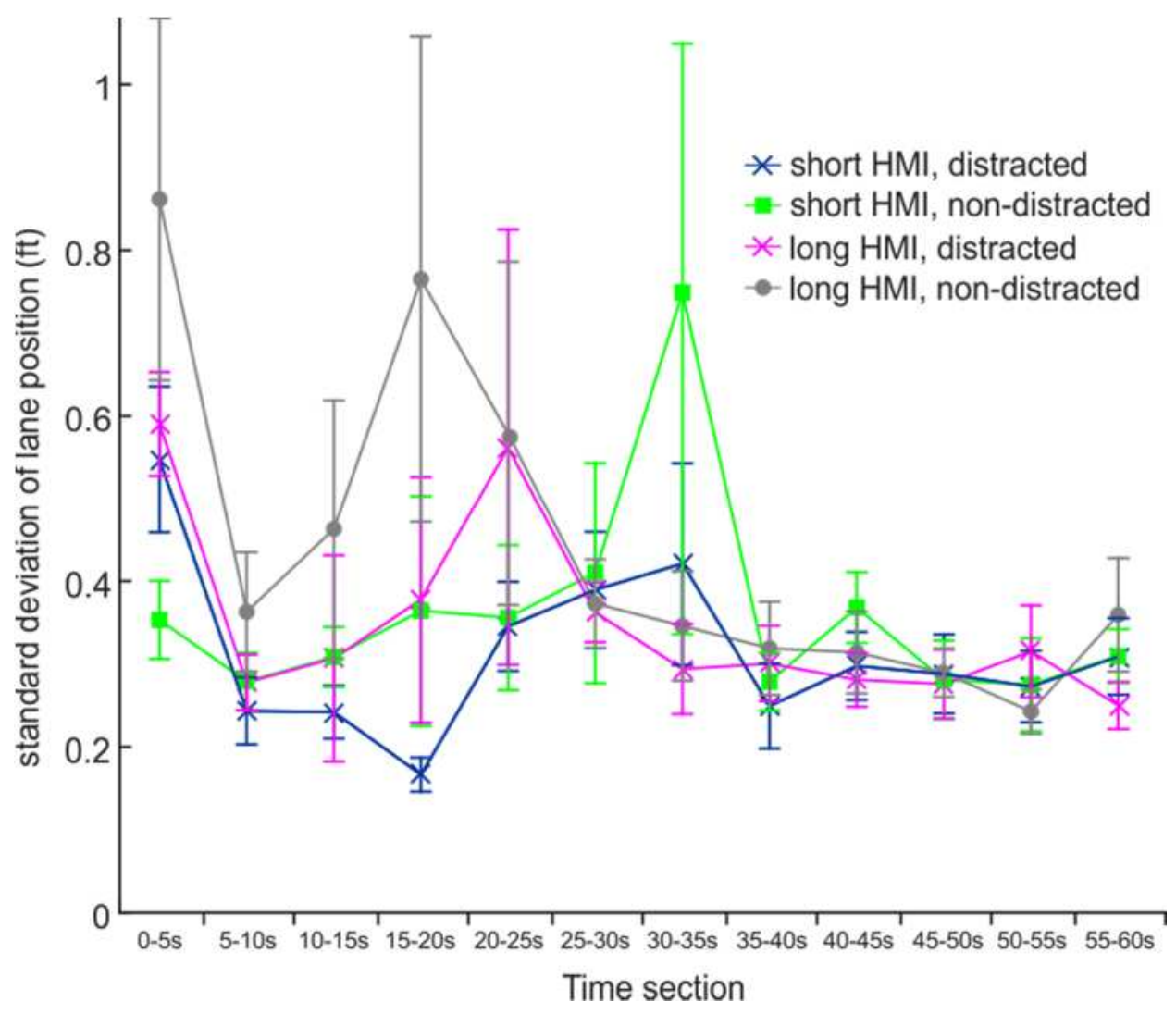

Figure 3

\section{Eye Tracking Measures}

\section{Mean Fixation Duration}

The analysis of the mean fixation's 5-second segments produced no significant main effect, e.g. for HMI $(\mathrm{p}=.392)$, Task $(\mathrm{p}=.060)$ and Time $(\mathrm{p}=.401)$. Similarly, the mean values of the first 60 seconds did not lead to any significant effects (HMI: $p=.329$, Task: $\mathrm{p}=.096, \mathrm{HMI}^{*}$ Task: $\mathrm{p}=.846$ ), although the, on average, mean fixation duration was longer with distraction task $(M=362.98, S D=259.99)$ than without $(M=309.34$, $\mathrm{SD}=148.84)$.

\section{Number of Fixations on HMI}

Friedman tests resulted in several significant effects. For the short HMI without 
distraction task, there was a main effect $\left[\chi^{2}(16)=114.042, p<.001, \varphi=2.670\right]$. Posthoc comparisons show that significant decreases occurred between 1-5 s and 5-10 $\mathrm{s}(\mathrm{p}=$ .036). For the short HMI with distraction task, there was a main effect as well $\left[\chi^{2}(16)=\right.$ 91.677, $\mathrm{p}<.001, \varphi=2.394]$, but no combination of adjacent sections was significantly different.

A main effect was identified for the long HMI without distraction task $\left[\chi^{2}(16)=\right.$ $108.548, \mathrm{p}<.001, \varphi=2.605]$, but this effect could not be attributed to any combination of adjacent sections. Also for the long HMI with distraction task, the main effect $\left[\chi^{2}(16)\right.$ $=108.295, \mathrm{p}<.001, \varphi=2.602]$ could not be found in the adjacent sections. The 60second means were normally distributed. On average, the short HMI attracted more fixations, $6.4(\mathrm{SD}=4.9)$ when not distracted and $4.5(\mathrm{SD}=3.8)$ when distracted, but there were no significant main effects (HMI: $\mathrm{p}=.088$, Task: $\mathrm{p}=.281$, HMI*Task: $\mathrm{p}>$ .999). The values for this measure are presented in Error! Reference source not found. 


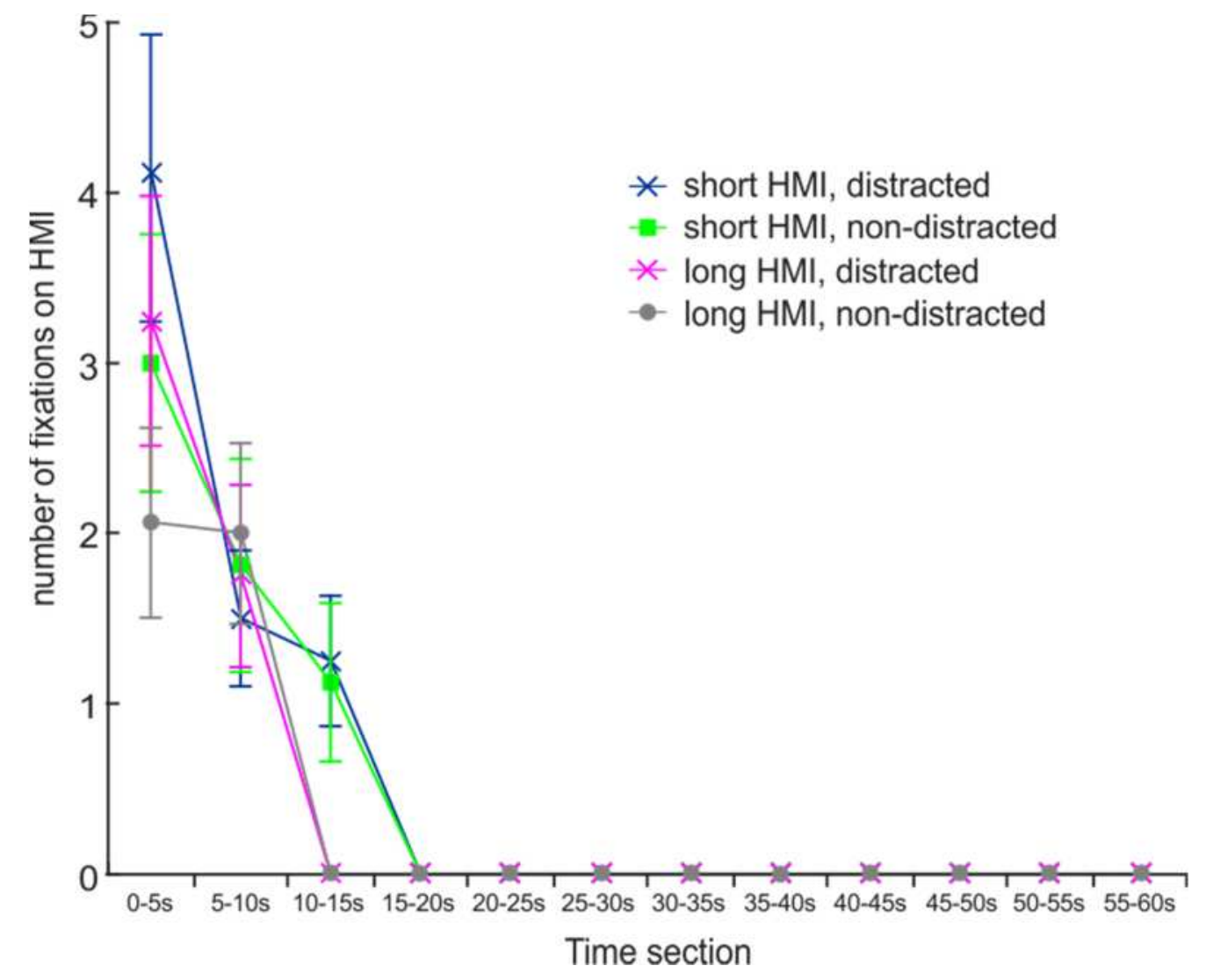

Figure 4

\section{Percentage Road Centre}

For the short HMI, there was no main effect without the distraction task $(\mathrm{p}=.804)$.

With the distraction task, a main effect was found $\left[\chi^{2}(15)=25.305, p=.008, \varphi=\right.$ 1.299], which was not confirmed with the 5-second bins. For the long HMI, no main effect occurred, either without $(\mathrm{p}=.653)$ or with the distraction task $(\mathrm{p}=.889)$. The 60 second means were normally distributed, and there were no significant main effects (HMI: $\mathrm{p}=.107$, Task: $\mathrm{p}=.361$, HMI*Task: $\mathrm{p}=.198)$

\section{Spread of Visual Search}

There was a main effect for Time $\left[F(11,143)=2.401, p=.009, \eta^{2}=.156\right]$. According to pairwise post-hoc comparisons, none of the adjacent sections were significantly different from each other. There were no effects for HMI $(p=.315)$, Task $(p=.393)$ 
and no interaction effects. For the 60 -second means, there were no significant main effects (HMI: $\mathrm{p}=.359$, Task: $\mathrm{p}=.317$, HMI*Task: $\mathrm{p}=.078)$.

\section{Discussion}

This study investigated the effects of long and short non-critical TOC requests on vehicle control and visual search behaviour. The study focused on the tactical and control levels of Michon's hierarchies of human performance as well as SA. It was hypothesised that the long TOC time of 50 seconds and the absence of a distraction task (tablet game) would lead to better adjustments to changed traffic conditions, more stable vehicle control, and more efficient visual scanning patterns, compared to short 5-second TOCs following level 3 automation with the distraction task.

As expected, tactical-level behaviours improved with a longer TOC time. In particular, drivers increased their speed sooner, measured by the minimum and mean speed. However, by the end of the first minute of manual driving, the differences between the HMI conditions had diminished. These findings suggest that, with lengthy TOC times, drivers were able to better prepare for speed increases and an adjustment to the changed motorway conditions, as the traffic jam dissolved. This finding is in line with previous studies, showing that longer TOC times can improve SA and decision making on the tactical level (Damböck et al. 2012). The eye tracking data show that the number of fixations on the HMI was highest, overall, within the first 5 seconds after handover. It then decreased towards the 10-second point, but this change was only significant for the short HMI condition without the distraction task. On average, people fixated more often on the HMI after the end of the short handover, although this difference was not significant, probably due to the high variation between participants. Overall, the short HMI might have created the need for more time to become aware of the system state in order to make behavioural decisions at the tactical level. Previous 
studies suggest similar needs to rebuild SA after automated periods, as drivers tend to delay reactions after handover situations, particularly when the road environment is complex or critical (Gold et al. 2016; Louw, Merat, and Jamson 2015). They also react in a more calculated manner after passively observing the road compared to engaging in a non-driving task (Eriksson and Stanton 2017b; Louw, Merat, and Jamson 2015). In addition, they are generally more willing to observe the environment during automation when it is more complex (Jamson et al. 2013).

Interestingly, there were no significant differences in the other eye tracking measures, mean fixation duration, percent road centre and spread of search. These measures constitute the efficiency of visual search, which tends to be inversely related to cognitive workload (e.g. Chapman and Underwood 1998; Konstantopoulos, Chapman, and Crundall 2010; Chapman et al. 2007) and traffic complexity (Victor 2005). It is possible that these measures refer to sensory input for the control level and thus do not change with the amount of tactical information processing. In fact, it has been shown that drivers can compensate for elevated workload with behaviours such as speed and headway (de Waard 1996; Horberry et al. 2006), but visual search patterns may be more difficult to consciously control (Recarte and Nunes 2003). Another possible explanation is that drivers may not need to alter these search strategies to gain their necessary SA. However, Louw et al. (2016) have shown that a TOC after a secondary task can lead to more erratic search behaviours, indicating more intensive visual search. Although their study included conditions with critical events and foggy environments, eye movements stabilised within one second. In contrast, the motorway scenario employed in the current study could have been simple and straightforward enough to allow appropriate skilled, subconscious visual search. 
The data present mixed results for several behavioural measures after engaging in the distracting game during the automation. Whether a distracting game was played was not reflected in effects for mean, minimum and the variation of speed. This suggests that drivers could have been engaged to some degree in monitoring the driving scene (cf. Jamson et al. 2013; Smith and Jamieson 2012) and thus not been fully OOTL when not playing the tablet game. Interestingly, participants were not increasing their lateral variability in the distracted conditions, regardless of whether the TOC was short or long. In contrast, when not distracted during the automation, drivers tended to spend several seconds stabilising their lane position, which took up to 25 seconds in the case of the short HMI. The game might have influenced some level of vehicle control positively, potentially by raising overall arousal and workload moderately. Wickens (2002) argues that elevated workload does not necessarily affect performance itself, but the potential of performing in the task. Similarly, the Malleable Attentional Resources Theory (MART, Young and Stanton 2002) states that the available attentional resource pool may change its size with the demands of the current task, and the game might have resulted in a larger attentional resource pool, which was then available for use in the subsequent driving task. An alternative explanation is provided by Madigan et al. (2018), who argued that increased lateral instability after level 3 automation may be due to the driver attempting to disengage the automation by turning the steering wheel. In the present study, drivers could have attempted this disengagement when not playing the game.

The results of the current study have several implications for the design of TOC following periods of level 3 automation. This study employed a longer TOC period, which was apparently effective for the driver to regain sufficient SA before manual driving, especially for tactical behaviours. In current level 2 and announced level 3 
systems, the driver gains control as soon as they provide an input to the steering wheel or pedals. Such a philosophy delegates the judgement of the readiness to takeover to the driver, which may affect vehicle control. In fact, Eriksson and Stanton (2017a) found that driver-paced transitions from automation to manual driving can lead to better lateral control. Alternative strategies may bear the risk that other supporting functions such as lane departure warnings might be necessary to avoid lateral instability. Table 2 lists such support systems, showing how either higher or lower levels of the driving task can be supported. A top-down approach would concentrate on the strategic and tactical levels informing the driver of potential navigation and manoeuvring decisions, for example. In contrast, a bottom-up approach would feed into the lower level, supporting the driver with haptic feedback and information about the immediate stabilisation of the car. These need to be further investigated in future studies.

Table 2 HMI design philosophies to support takeovers from top-down and bottom-up approaches

\begin{tabular}{|l|l|}
\hline HMI design philosophy & Supporting functions \\
\hline Top-down & $\begin{array}{l}\text { Navigation information and route planning } \\
\text { Real-time information on traffic and route conditions } \\
\text { Manoeuvre suggestions and support, e.g. blind spot } \\
\text { warnings }\end{array}$ \\
\hline Bottom-up & $\begin{array}{l}\text { ACC } \\
\text { Lane departure warning } \\
\text { Haptic feedback }\end{array}$ \\
\hline
\end{tabular}

The study has several limitations. In particular, further conditions with different TOC times could be added to investigate the measures as well as the drivers' judgement of their readiness in more detail, as the current measures and statistical effects do not allow 
understanding for the entirety of the effects. The study used a sample of 16 participants, a number that is in line with other driving simulator studies (Rapoport and Baniña 2007), but statistical power can be increased with a larger sample. In addition, realworld studies would remove the safety of the driving simulator and thus elicit more realistic responses. Such studies could be conducted with longer automation periods, a factor that has been shown to increase OOTL problems (Molloy and Parasuraman 1996), as well as the absence of experimenter instructions, which could prepare participants for a potential TOC situation. A particularly interesting research field within novel vehicle systems are novice drivers, a demographic that might increase in numbers in a future in which people drive less manually. Novice drivers tend to display inefficient visual search behaviour (Chapman and Underwood 1998; Crundall and Underwood 1998; Konstantopoulos, Chapman, and Crundall 2010), possibly affecting visual search during and after automation periods. Plus, as their mental workload during manual driving is higher, the implications of the MART and thus raised mental workload through a game might not improve lateral stability for them (cf. Young and Stanton 2007).

\section{Conclusions}

The study aimed to investigate the effects of long and short TOC requests on vehicle control and visual search on the tactical and on the control levels, and the impact of a distracting secondary task (game on a tablet computer) on these measures. The results suggest faster and more stable longitudinal vehicle control as well as higher SA following the long TOC request. Where drivers were not subjected to the distracting game, similar performance improvements could be observed. However, in the nondistracted conditions, drivers also exhibited worse lateral performance during the first 10 seconds of manual driving. Differences for visual search were not significant. This 
suggests that drivers may be willing to maintain some degree of SA maintenance during level 3 automation, or may remain more cognitively ready to drive when performing the game.

The study provides several discussion points for the implications for TOC strategies in level 3 automation, particularly the information provision of the HMI during the TOC, especially with regards to Rasmussen's and Michon's hierarchical levels of the driving task. The authors suggest to support the driving task at all hierarchical levels to aid rebuilding appropriate SA. In addition, future research needs to further investigate vehicle- as well as driver-initiated takeovers to test when the driver judges themselves as ready to drive manually.

Acknowledgements: The research was conducted in collaboration with Jaguar LandRover Research and the authors gratefully acknowledge their support.

\section{References}

Banks, Victoria A., Alexander Eriksson, Jim O'Donoghue, and Neville A. Stanton. 2018. "Is partially automated driving a bad idea? Observations from an on-road study." Applied Ergonomics 68 (Supplement C):138-45. doi: https://doi.org/10.1016/j.apergo.2017.11.010.

Beller, Johannes, Matthias Heesen, and Mark Vollrath. 2013. "Improving the DriverAutomation Interaction: An Approach Using Automation Uncertainty." Human Factors 55 (6):1130-41. doi: 10.1177/0018720813482327.

Boer, E.R., and M. Hoedemaeker. 1998. "Modeling driver behavior with different degrees of automation: A hierarchical decision framework of interacting mental models." In Conference on human decision making and manual control, edited by Patrick Millot. Valenciennes: University.

Chapman, P., E. van Loon, S. Trawley, and D. Crundall. 2007. "A comparison of drivers' eye movements in filmed and simulated dangerous driving situations." In Behavioural research in road safety, 2007: Seventeenth seminar, 112-23. London, UK: Department for Transport.

Chapman, Peter R , and Geoffrey Underwood. 1998. "Visual Search of Driving Situations: Danger and Experience." Perception 27 (8):951-64. doi: doi:10.1068/p270951. 
Crundall, David E., and Geoffrey Underwood. 1998. "Effects of experience and processing demands on visual information acquisition in drivers." Ergonomics 41 (4):448-58. doi: 10.1080/001401398186937.

Damböck, Daniel, Klaus Bengler, M Farid, and L Tönert. 2012. "Übernahmezeiten beim hochautomatisierten Fahren." In Tagung Fahrerassistenz, edited by Markus Lienkamp. München, Germany: Lehrstuhl für Fahrzeugtechnik (FTM).

de Waard, Dick. 1996. The measurement of drivers' mental workload, Dissertation. The Netherlands: Rijks Universiteit Groningen, Traffic Research Center.

Dekker, Sidney W. A. 2015. "The danger of losing situation awareness." Cognition, Technology \& Work 17 (2):159-61. doi: 10.1007/s10111-015-0320-8.

Endsley, Mica R. . 1995. "Toward a Theory of Situation Awareness in Dynamic Systems." Human Factors 37 (1):32-64. doi: 10.1518/001872095779049543.

Endsley, Mica R., and Esin O. Kiris. 1995. "The Out-of-the-Loop Performance Problem and Level of Control in Automation." Human Factors 37 (2):381-94. doi: $10.1518 / 001872095779064555$.

Eriksson, Alexander, and Neville A. Stanton. 2017a. "Driving Performance After SelfRegulated Control Transitions in Highly Automated Vehicles." Human Factors 59 (8):1233-48. doi: 10.1177/0018720817728774.

. 2017b. "Takeover Time in Highly Automated Vehicles: Noncritical Transitions to and From Manual Control." Human Factors 59 (4):689-705. doi: 10.1177/0018720816685832.

Gold, Christian, Moritz Körber, David Lechner, and Klaus Bengler. 2016. "Taking Over Control From Highly Automated Vehicles in Complex Traffic Situations." Human Factors 58 (4):642-52. doi: doi:10.1177/0018720816634226.

Goodrich, M. A., and E. R. Boer. 2003. "Model-based human-centered task automation: a case study in ACC system design." Systems, Man and Cybernetics, Part A: Systems and Humans, IEEE Transactions on 33 (3):325-36. doi: 10.1109/tsmca.2003.817040.

Horberry, Tim, Janet Anderson, Michael A. Regan, Thomas J. Triggs, and John Brown. 2006. "Driver distraction: The effects of concurrent in-vehicle tasks, road environment complexity and age on driving performance." Accident Analysis \& Prevention 38 (1):185-91. doi: http://dx.doi.org/10.1016/j.aap.2005.09.007.

Jamson, A. Hamish, Natasha Merat, Oliver M. J. Carsten, and Frank C. H. Lai. 2013. "Behavioural changes in drivers experiencing highly-automated vehicle control in varying traffic conditions." Transportation Research Part C: Emerging Technologies 30 (Supplement C):116-25. doi: https://doi.org/10.1016/j.trc.2013.02.008.

Kircher, K. , A. Larsson, and J. A. Hultgren. 2014. "Tactical Driving Behavior With Different Levels of Automation." IEEE Transactions on Intelligent Transportation Systems 15 (1):158-67. doi: 10.1109/TITS.2013.2277725.

Konstantopoulos, Panos, Peter Chapman, and David Crundall. 2010. "Driver's visual attention as a function of driving experience and visibility. Using a driving simulator to explore drivers' eye movements in day, night and rain driving." Accident Analysis \& Prevention 42 (3):827-34. doi: http://dx.doi.org/10.1016/j.aap.2009.09.022.

Louw, T., R. Madigan, O. Carsten, and N. Merat. 2016. "Were they in the loop during automated driving? Links between visual attention and crash potential." Injury Prevention 23 (4):281-6.

Louw, Tyron, Natasha Merat, and Hamish Jamson. 2015. "Engaging with highly automated driving: to be or not to be in the loop." In Proceedings of the Eighth 
International Driving Symposium on Human Factors in Driver Assessment, Training and Vehicle Design Salt Lake City, UT: University of Iowa.

Madigan, Ruth, Tyron Louw, and Natasha Merat. 2018. "The effect of varying levels of vehicle automation on drivers' lane changing behaviour." PLOS ONE 13 (2):e0192190. doi: 10.1371/journal.pone.0192190.

Matthews, Michael, David Bryant, Robert Webb, and Joanne Harbluk. 2001. "Model for Situation Awareness and Driving: Application to Analysis and Research for Intelligent Transportation Systems." Transportation Research Record: Journal of the Transportation Research Board 1779 (1):26-32. doi: 10.3141/1779-04.

Merat, Natasha, A. Hamish Jamson, Frank C. H. Lai, Michael Daly, and Oliver M. J. Carsten. 2014. "Transition to manual: Driver behaviour when resuming control from a highly automated vehicle." Transportation Research Part F: Traffic Psychology and Behaviour 27, Part B:274-82. doi: http://dx.doi.org/10.1016/j.trf.2014.09.005.

Michon, J.A. 1985. "A critical view of driver behavior models: What do we know, what should we do." In Human behavior and traffic safety, edited by Leonard Evans and Richard C. Schwing, 485-520. New York, NY: Plenum.

Molloy, Robert, and Raja Parasuraman. 1996. "Monitoring an Automated System for a Single Failure: Vigilance and Task Complexity Effects." Human Factors: The Journal of the Human Factors and Ergonomics Society 38 (2):311-22. doi: 10.1177/001872089606380211.

Naujoks, Frederik, Christoph Mai, and Alexandra Neukum. 2014. "The effect of urgency of take-over requests during highly automated driving under distraction conditions." In Advances in Human Aspects of Transportation, edited by Neville A. Stanton, Giuseppe Di Bucchianico, Andrea Vallicelli and Steven Landry, 431-8. Krakow, Poland: AHFE Conference.

NHTSA. PE 16-007. "ODI Resume." https://static.nhtsa.gov/odi/inv/2016/INCLAPE16007-7876.PDF.

Rapoport, Mark J., and Melanie C. Baniña. 2007. "Impact of Psychotropic Medications on Simulated Driving." CNS Drugs 21 (6):503-19. doi: 10.2165/00023210200721060-00006.

Rasmussen, J. 1983. "Skills, rules, and knowledge; signals, signs, and symbols, and other distinctions in human performance models." Systems, Man and Cybernetics, IEEE Transactions on SMC-13 (3):257-66. doi: 10.1109/tsmc.1983.6313160.

Recarte, Miguel A., and Luis M. Nunes. 2003. "Mental workload while driving: Effects on visual search, discrimination, and decision making." Journal of Experimental Psychology: Applied 9 (2):119-37. doi: 10.1037/1076898X.9.2.119.

Roncoli, Claudio, Markos Papageorgiou, and Ioannis Papamichail. 2015. "Traffic flow optimisation in presence of vehicle automation and communication systems Part I: A first-order multi-lane model for motorway traffic." Transportation Research Part C: Emerging Technologies 57:241-59. doi: https://doi.org/10.1016/j.trc.2015.06.014.

Salmon, Paul M., Guy H. Walker, and Neville A. Stanton. 2016. "Pilot error versus sociotechnical systems failure: a distributed situation awareness analysis of Air France 447." Theoretical Issues in Ergonomics Science 17 (1):64-79. doi: 10.1080/1463922X.2015.1106618. 
Seppelt, Bobbie D., and John D. Lee. 2007. "Making adaptive cruise control (ACC) limits visible." International Journal of Human-Computer Studies 65 (3):192205. doi: 10.1016/j.ijhcs.2006.10.001.

Smith, Adam G. , and Greg A. Jamieson. 2012. "Level of Automation Effects on Situation Awareness and Functional Specificity in Automation Reliance." In Proceedings of the Human Factors and Ergonomics Society Annual Meeting, 2113-7. Boston, MA: Human Factors and Ergonomics Society.

Society of Automotive Engineers. 2014. "Taxonomy and Definitions for Terms Related to On-Road Motor Vehicle Automated Driving Systems." In. Hong Kong, China.

van der Heiden, Remo M.A., Shamsi T. Iqbal, and Christian P. Janssen. 2017. "Priming Drivers before Handover in Semi-Autonomous Cars." In Proceedings of the 2017 CHI Conference on Human Factors in Computing Systems, edited by Caroline Appert, Daniel Wigdor and Juan Pablo Hourcade, 392-404. Denver, Colorado, USA: ACM.

Victor, Trent. 2005. "Keeping Eye and Mind on the Road." Doctoral thesis, comprehensive summary, Uppsala University.

Victor, Trent W., Joanne L. Harbluk, and Johan A. Engström. 2005. "Sensitivity of eyemovement measures to in-vehicle task difficulty." Transportation Research Part F: Traffic Psychology and Behaviour 8 (2):167-90. doi: http://dx.doi.org/10.1016/j.trf.2005.04.014.

Ward, Nicholas J. 2000. "Automation of task processes: An example of intelligent transportation systems." Human Factors and Ergonomics in Manufacturing \& Service Industries 10 (4):395-408.

Wickens, Christopher D. 2002. "Multiple resources and performance prediction." Theoretical Issues in Ergonomics Science 3 (2):159-77. doi: 10.1080/14639220210123806.

Young, M. S., and N. A. Stanton. 2007. "What's skill got to do with it? Vehicle automation and driver mental workload." Ergonomics 50 (8):1324-39. doi: 10.1080/00140130701318855.

Young, Mark S. , and Neville A. Stanton. 2002. "Malleable Attentional Resources Theory: A New Explanation for the Effects of Mental Underload on Performance." Human Factors 44 (3):365-75. doi: doi:10.1518/0018720024497709.

Zeeb, Kathrin, Axel Buchner, and Michael Schrauf. 2016. "Is take-over time all that matters? The impact of visual-cognitive load on driver take-over quality after conditionally automated driving." Accident Analysis \& Prevention 92 (Supplement C):230-9. doi: https://doi.org/10.1016/j.aap.2016.04.002. 